\title{
STABILITY OF POLLING SYSTEMS WITH EXHAUSTIVE SERVICE POLICIES AND STATE-DEPENDENT ROUTING
}

\author{
By Serguei Foss ${ }^{1}$ and GÜNTER LAST
}

\author{
Novosibirsk State University and Technical University \\ of Braunschweig
}

\begin{abstract}
We consider a polling system with a finite number of stations fed by compound Poisson arrival streams of customers asking for service. A server travels through the system and upon arrival at a station the server serves all waiting customers until the queue is empty, where the service time distribution depends on the station. The choice of the station to be visited next as well as the corresponding walking time may depend on the whole current state. Examples are systems with a greedy-type routing mechanism. Under appropriate independence assumptions it is proved that the system is stable if and only if the workload is less than 1.
\end{abstract}

1. Introduction. Consider a server who visits ( polls) the stations of a queueing network. The stations are numbered 1 through $K$ and with each of them there is associated a queue with infinite waiting capacity fed by an arrival stream of customers with intensity $\lambda_{i}, i=1, \ldots, K$. The process of all arrival instants is assumed to be homogeneous Poisson. At a given arrival instant, however, all stations may simultaneously receive a group of customers. The joint distribution of these groups should render the expected group sizes positive and finite. The server employs the so-called exhaustive service policy at each station. This means that, upon arriving at a nonempty station, he will provide service there until the moment when the station becomes empty, which includes service for all those customers who may arrive during the service of the customers present at the time of the server's arrival. The services are independent of the arrival stream and each served customer departs from the system. The service times at station $i$ are i.i.d. and are assumed to have a finite positive mean $b_{i}$. When the server has finished the batch of services at station $i$ or if he has found that station empty, then he walks to another station $j$, say, taking a walking time that might also be 0 . The choice of this station and the associated walking time depend on the whole current state of the system and may even depend on future arrivals. An example is the greedy routing mechanism, where the server chooses a station with the maximum number of customers in a certain neighborhood waiting at the start of the walk.

\footnotetext{
Received January 1995; revised September 1995.

${ }^{1}$ This work was done while the first author held a visiting position at the Technical University of Braunschweig. Partial support was provided by INTAS Grant 93-820.

AMS 1991 subject classifications. Primary 60K25; secondary 60J27.

Key words and phrases. Polling system, stability, ergodicity of Markov chains, greedy server.
} 
The aim of the present paper is to establish a stability condition for the queueing network ensuring that the server can handle all the work arriving at the network. Mathematically, this amounts to proving the ergodicity of the underlying Markov chain. Under appropriate independence assumptions it will be shown that the system is stable if and only if

$$
\sum_{i=1}^{K} \lambda_{i} b_{i}<1
$$

It is worth mentioning that our stability results can be proved under more general i.i.d. assumptions on the input using the same ideas. We have decided to work under the Poisson assumption in order to avoid technicalities which could obscure the main arguments.

A special feature of the present model is that the server's decision about which station to serve next depends on the actual configuration of customers in the system. In Coffman and Gilbert (1987) one can find a discussion of a polling system with a greedy server on a circle and a line as well as a conjecture about the stability condition. The only earlier paper we are aware of, however, which solves the stability problem for polling systems with state-dependent routing is Schassberger (1993), where the ergodicity of a symmetric ringlike network with a limited service policy has been proved. These results are generalized in Foss and Last (1995) which deals with polling systems with a special greedy routing mechanism on a graph but with rather general service policies for each station. In this work conditions sufficient for stability and sufficient for instability are presented. These conditions coincide only in special (symmetric) cases and it is shown by examples that the determination of the exact stability region might be a difficult task. There are many other papers establishing comparison and stability results for polling systems with state-independent routing. We refer here to Levy, Sidi and Boxma (1990), Georgiadis and Szpankowski (1992), Borovkov and Schassberger (1994) and Fricker and Jaibi (1994). Massoulié (1995) constructed a stationary regime for polling systems with stationary ergodic arrival process and with state-independent routing mechanism. Thereafter, for a more general model, Foss and Chernova (1996) proved the stability results using the saturation rule of Baccelli and Foss (1995). In this context one should also mention the papers by Kroese and Schmidt (1992, 1994) proving the stability of a polling model on the circle and by Altman and Levy (1994) on polling systems in space. For applications of polling models we refer to Coffman and Gilbert (1987) and Takagi (1990), who gives a survey of the extensive literature.

The idea of the stability proof in this paper is to construct, by induction on the number of stations, a stopping time at which a certain linear test function satisfies a multiplicative drift condition. Ergodicity is then obtained from a general stability result on Markov chains which is a "randomized" version of stability criteria by Malyshev and Menshikov (1982); see also Meyn and Tweedie (1993, 1994), Borovkov (1994), Dai (1995) and Fayolle, Malyshev and Menshikov (1995). 
The paper is organized as follows. Section 2 gives the extensive and exact description of the model and introduces some notation. Section 3 presents the proof of the stability result. The Appendix contains some general results for discrete-time Markov processes needed in the course of our paper.

2. Model description. We consider a queueing system consisting of $K$ stations with infinite waiting capacities. With each station there is associated a queue of customers waiting for service. One server is traveling through the system. Upon arrival of the server at a station, the corresponding queue is served until it is empty, including customers arriving after the server's arrival instant. Each service takes a random time and each served customer leaves the system.

We let $X_{i}(t)$ denote the number of customers in the $i$ th queue at time $t \in \mathbb{R}_{+}$. Further we denote by $S(t)$ the number of the station which is occupied by the server while serving is in progress and we let $S(t):=0$ otherwise. Both $X_{i}(t)$ and $S(t)$ are taken to be right continuous, and we can assume that there exist limits from the left, denoted by $X_{i}(t-)$ and $S(t-)$.

Let $T_{n}, n \in \mathbb{N}$, be the time of the $n$th service completion and $T^{n+1}$ the time of the beginning of the next service after time $T_{n}$. If $X_{S\left(T_{n}-\right)}\left(T_{n}\right)>0$, then $T_{n}=T^{n+1}$. If $X_{S\left(T_{n}-\right)}\left(T_{n}\right)=0$, then

$$
W_{n+1}:=T^{n+1}-T_{n}
$$

is the walking time taken by the server to travel from station $S\left(T_{n}-\right)$ to $S\left(T^{n+1}\right)=S\left(T_{n+1}-\right)$. The cases $W_{n+1}=0$ and $S\left(T_{n}-\right)=S\left(T^{n+1}\right)$ are not excluded. It should be kept in mind that the server may have visited several empty stations before he starts serving queue $S\left(T^{n+1}\right)$. Hence in certain cases the time $W_{n+1}$ can be regarded as a sum of walking times. If the system is empty at time $T_{n}$, then a part of $W_{n+1}$ is, in fact, used to wait for the next arriving customer. Hence $W_{n+1}$ has to be positive in that case. We refer here to the model description below as well as to Examples 2.1 and 2.2.

The initial conditions are given by a random element $X(0)=$ $\left(X_{1}(0), \ldots, X_{K}(0), S_{0}\right)$ of $\mathbb{Z}_{+}^{K} \times\{1, \ldots, K\}$. If $X_{S_{0}}(0)=0$, then the server starts walking as described above. In this case $T^{1}=W_{1}$ is defined as the time of the beginning of the first service, and we set $S(0-):=S_{0}$ and $S(t)=0$ for $0 \leq t<T^{1}$. [If $T^{1}=0$ we have $S(0)=S\left(T^{1}\right)$.] If $X_{S_{0}}(0)>0$, then the server starts serving until the queue $S(0-)=S(0):=S_{0}$ is empty. In this case we define $W_{1}=T^{1}:=0$.

In order to explain how the system is operating, we will now describe the arrival processes, the services and the assumptions on the routing and walking times. The underlying probability space is denoted by $(\Omega, \mathscr{F}, P)$. Define

$$
\mathscr{F}_{t}:=\sigma(X(s): s \leq t), \quad t \in \mathbb{R}_{+},
$$

where $X(t):=\left(X_{1}(t), \ldots, X_{K}(t), S(t)\right)$. Then $\left\{\mathscr{F}_{t}: t \in \mathbb{R}_{+}\right\}$is a right-continuous filtration describing the internal history of the process. We let $A_{i}(t)$ denote 
the number of customers who have arrived at station $i$ by time $t$. Hence

$$
A_{i}(s, t]:=A_{i}(t)-A_{i}(s), \quad s \leq t,
$$

is the number of customers who arrived at station $i$ during the time interval $(s, t]$. We assume that the arrival instants are different from the times of completion of services so that

$$
A_{i}(t)=\sum_{s: X_{i}(s)>X_{i}(s-)} \mathbf{1}\{s \leq t\}\left(X_{i}(s)-X_{i}(s-)\right), \quad t \in \mathbb{R}_{+},
$$

is $\mathscr{F}_{t}$-measurable. We also note that

$$
T_{n+1}=\min \left\{t \geq T_{n}: \sum_{i=1}^{K} X_{i}(t)<\sum_{i=1}^{K} X_{i}(t-)\right\}, \quad n \in \mathbb{Z}_{+},
$$

where $T_{0}:=0$, and

$$
T^{n+1}=\inf \left\{t \geq T_{n}: S(t) \neq 0\right\}, \quad n \in \mathbb{Z}_{+} .
$$

Hence $T_{n}$ and $T^{n}, n \in \mathbb{N}$, are $\left\{\mathscr{F}_{t}\right\}$-stopping times. If $T$ is any $\left\{\mathscr{F}_{t}\right\}$-stopping time, then, as usual,

$$
\mathscr{F}_{T}:=\left\{A \in \mathscr{F}_{\infty}: A \cap\{T \leq t\} \in \mathscr{F}_{t} \text { for all } t \in \mathbb{R}_{+}\right\}
$$

denotes the history at time $T$, where $\mathscr{F}_{\infty}:=\sigma\left(\bigcup_{s>0} \mathscr{F}_{s}\right)$.

The sequence $\left(\tau_{n}\right)$ of all arrival instants is assumed to form a homogeneous Poisson process with intensity $\lambda$. Further there is given a sequence $\left(B_{n}\right)=$ $\left(\left(B_{n}^{1}, \ldots, B_{n}^{K}\right)\right)$ of independent random elements of $\mathbb{Z}_{+}^{K}$ which is independent of $\left(\tau_{n}\right)$ and such that $a_{i}:=E B_{1}^{i}$ is positive and finite for all $i$ and $\sum_{i} B_{n}^{i}>0$. The arrival process $A_{i}(t)$ is then assumed to be given by

$$
A_{i}(t):=\sum_{n \geq 1} \mathbf{1}\left\{\tau_{n} \leq t\right\} B_{n}^{i} .
$$

It is a compound Poisson process with intensity $\lambda_{i}:=\lambda a_{i}$. Note that the $\left\{A_{i}(t)\right\}$ are independent if and only if the components of $B_{1}$ are independent. We will assume more generally that $\left(\left(\tau_{n}, B_{n}\right)\right)$ is a marked $\left\{\mathscr{F}_{t}\right\}$-Poisson process; see, for example, Last and Brandt (1995). That is to say that

$$
\sum_{n \geq 1} \mathbf{1}\left\{s<\tau_{n} \leq t, B_{n} \in C\right\}
$$

is independent of $\mathscr{F}_{s}$ for all $s<t$ and all measurable $C \subseteq \mathbb{Z}_{+}^{K}$. In particular, $A_{i}(t, v]$ is independent of $\mathscr{F}_{t}$ for all $t<v$. This property generalizes to an $\left\{\mathscr{F}_{t}\right\}$-stopping time $T$; that is, $A_{i}(T, v]$ is independent of $\mathscr{F}_{T}$ for all $v$, where $A_{i}((T, v])=0$ if $v \leq T$. Also,

$$
E A_{i}(T)=\lambda_{i} E T, \quad i \in\{1, \ldots, K\},
$$

for any $\left\{\mathscr{F}_{t}\right\}$-stopping time $T$. More generally, if $S$ is another $\left\{\mathscr{F}_{t}\right\}$-stopping time satisfying $S \leq T$, then

$$
E\left[A_{i}(S, T] \mid \mathscr{F}_{S}\right]=\lambda_{i} E\left[T-S \mid \mathscr{F}_{S}\right], \quad P \text {-a.s., } i \in\{1, \ldots, K\} .
$$


We assume that service times are independent of the arrival process and that

$$
P\left(T_{n}-T^{n} \in \cdot \mid \mathscr{F}_{T^{n}}\right)=G_{i}(\cdot), \quad P \text {-a.s. on }\left\{S\left(T^{n}\right)=i\right\}, n \in \mathbb{N},
$$

where $G_{1}, \ldots, G_{K}$ are distributions on $(0, \infty)$ with finite and positive means $b_{1}, \ldots, b_{K}$. In particular, different service times are independent.

Assume that $S\left(T_{n}-\right)=i, n \in \mathbb{N}$, and $X_{i}\left(T_{n}\right)=0$. Then the server stops serving station $i$ and the station $S\left(T^{n+1}\right)=j$ to be served next satisfies $X_{j}\left(T^{n+1}\right)>0$. The choice of the station $j$ and the random variable $W_{n+1}$ may depend on the whole actual state of the system and even on the arrivals after time $T_{n}$, and the corresponding conditional distributions might be quite general; see Examples 2.1 and 2.2. However, we assume that there is a finite constant $w$ such that

$$
E\left[W_{1} \mid \mathscr{F}_{0}\right] \leq w, \quad P \text {-a.s. on }\left\{X_{S_{0}}(0)=0\right\},
$$

as well as a constant $p>0$ satisfying

$$
P\left(A\left(W_{1}\right)=0 \mid \mathscr{F}_{0}\right)>p, \quad P \text {-a.s. on }\left\{X_{S_{0}}(0)=0, \sum_{k} X_{k}(0)>0\right\},
$$

where

$$
A(t):=\sum_{i} A_{i}(t)
$$

The latter condition excludes some strange behavior of the server and seems to be satisfied in all relevant examples. It states that given the server has just completed a batch of services and the system is nonempty, then the probability that the next batch of services starts at one of these nonempty stations is uniformly bounded from below by a positive constant.

Our assumptions imply that the $T_{n}$ are almost surely finite and satisfy $\lim _{n \rightarrow \infty} T_{n}=\infty$ and hence we assume these properties to hold everywhere on $\Omega$. Denote

$$
\hat{S}(n):=S\left(T_{n}-\right), \quad n \in \mathbb{Z}_{+},
$$

where we recall that $T_{0}=0$; see also the discussion of the initial values above. The process

$$
\hat{X}(n):=\left(X_{1}\left(T_{n}\right), \ldots, X_{K}\left(T_{n}\right), \hat{S}(n)\right), \quad \hat{\mathscr{F}}_{n}:=\mathscr{F}_{T_{n}}, \quad n \in \mathbb{Z}_{+},
$$

is assumed to be a homogeneous Markov chain, where we dispense with making this assumption more explicit. This could be done by introducing appropriate kernels describing the conditional distribution of $\left(W_{n+1}\right.$, $\left.X_{1}\left(T^{n+1}\right), \ldots, X_{K}\left(T^{n+1}\right), S\left(T^{n+1}\right)\right)$ given $\mathscr{F}_{T_{n}}$ and $X_{S\left(T_{n}-\right)}\left(T_{n}\right)=0$. Because we do not need these kernels later on, we prefer to illustrate our model by examples.

EXAMPLE 2.1. Assume that for each $i \in\{1, \ldots, K\}$ there is a set $N(i) \subseteq$ $\{1, \ldots, K\}$ of neighbors of $i$ such that $i \in N(i)$. Assume that for all $i, j \in$ 
$\{1, \ldots, K\}$ there is a sequence $k_{1}, \ldots, k_{r} \in\{1, \ldots, K\}$ such that $k_{1}=i, k_{r}=j$ and $k_{m+1} \in N\left(k_{m}\right)$ for $1 \leq m \leq r-1$. This means that the neighborhood relation equips $\{1, \ldots, K\}$ with the structure of a directed and connected graph. Assume that $S\left(T_{n}-\right)=i, n \in \mathbb{N}$, and $X_{i}\left(T_{n}\right)=0$. Then the server stops serving station $i$ and selects another station $S\left(T^{n+1}\right)=j$, say, in such a way that $j \in N(i)$ and

$$
X_{j}\left(T_{n}\right)>0 \text { if } \sum_{k \in N(i)} X_{k}\left(T_{n}\right)>0,
$$

which means that the server walks to one of the nonempty stations in his neighborhood. This is a greedy-type routing mechanism. To describe the dynamics of this system more accurately, we assume that for any $x=$ $\left(x_{1}, \ldots, x_{K}, i\right) \in \mathbb{Z}_{+}^{K} \times\{1, \ldots, K\}$ there is a set $D_{x} \subseteq C_{x}:=\left\{j \in N(i): x_{j}>0\right\}$ such that $D_{x} \neq \varnothing$ whenever $C_{x} \neq \varnothing$. If $X(0)=x$ and $C_{x} \neq \varnothing$, then

$$
\begin{aligned}
& P\left(W_{1} \in d w, X\left(W_{1}\right)=y_{1}, \ldots, X\left(W_{K}\right)=y_{K}, S\left(W_{1}\right)=j \mid \mathscr{F}_{0}\right) \\
& =F_{i j}^{\prime}(d w) P\left(\left(x_{1}+A_{1}(w), \ldots, x_{K}+A_{K}(w)\right)=\left(y_{1}, \ldots, y_{K}\right)\right) \\
& \quad \times \mathbf{1}\left\{j \in D_{x}\right\} \frac{1}{\operatorname{card} D_{x}},
\end{aligned}
$$

where $F_{i j}^{\prime}$ is a distribution with a finite mean $w_{i j}$. Assume now that the system starts at time 0 in a state $x=\left(x_{1}, \ldots, x_{K}, i\right)$ satisfying $\sum_{k \in N(i)} x_{k}=0$. Then the server chooses a station $j$, say, randomly among the neighbors of $i$. Again it takes the server a random time $W^{1}$ with the distribution $F_{i j}(x)$ to walk from $i$ to $j$. If $X_{j}\left(W^{1}\right)>0$, then we put $W_{1}:=W^{1}$. If not, then the server chooses another station randomly in the set $A(j)$ of all stations $k \in N(j)$ satisfying $X_{k}\left(W^{1}\right)>0$, if there is such a station. If $\sum_{k \in N(j)} X_{k}\left(W^{1}\right)=0$, then $A(j):=N(j)$. The server continues in this way until he arrives at a nonempty station. Assume that $\inf _{x} \sum_{i, j} w_{i j}(x)>0$, where $w_{i j}(x)$ is the mean of $F_{i j}(x)$. Under obvious independence assumptions it takes the server a finite random time $W_{1}$ to arrive at a nonempty station. The process satisfies (2.3) and (2.4). We conclude this example by specifying possible choices of the sets $D_{x}$.

(i) Assume that

$$
D_{x}=\left\{j \in N(i): x_{j}=\max \left\{x_{k}: k \in N(i)\right\}\right\} .
$$

This defines the so-called greedy walking mechanism.

(ii) Assume that there are positive and finite numbers $d(i, j)$ indicating a distance between $j \in N(i)$ and $i$ and that

$$
D_{x}=\left\{j \in N(i): d(i, j)=\min \left\{d(i, k): k \in N(i), x_{k}>0\right\}\right\},
$$

where $\min \varnothing:=0$. In this case the server tries to walk to the nearest nonempty station in his neighborhood.

(iii) $D_{x}=\left\{j \in N(i): x_{j}>0\right\}$.

(iv) $D_{x}=\left\{j \in N(i): x_{j}=\min \left\{x_{k}: x_{k}>0\right\}\right\}$. 
(v) Let $0<c<d$ and assume that $x=\left(x_{1}, \ldots, x_{k}, i\right)$ satisfies $\sum_{k \in N(i)} x_{k}>0$. Let

$$
D_{x}=\left\{j \in N(i): c \leq x_{j} \leq d\right\}
$$

if this set is nonempty and let $D_{x}$ be given as in (i) otherwise.

We give now another example for a routing mechanism which was considered by Schassberger (1993) in the special case of a ringlike graph. In that case the polling system could be considered as an approximation of the greedy server on the circle.

EXAmple 2.2. Consider the situation of Example 2.1(ii). An $r$-tuple $p=$ $\left(k_{1}, \ldots, k_{r}\right) \in\{1, \ldots, K\}^{r}$ is called a path if $k_{m+1} \in N\left(k_{m}\right)$ for $1 \leq m \leq r-1$ and if all $k_{m}$ are distinct. The length $d(p)$ of such a path is defined by $d\left(k_{1}, k_{2}\right)+\cdots+d\left(k_{m-1}, k_{m}\right)$. Assume that the system starts at time 0 in state $x=\left(x_{1}, \ldots, x_{K}, i\right)$ satisfying $\sum_{k} x_{k}>0$. Consider the set of all paths $p$ starting at $i$ and ending at a station $k$ with $x_{k}>0$, and let $H_{x}$ be the subset where $d(p)$ becomes minimal. The server then chooses the next station $j$, say, randomly in the set

$$
D_{x}^{\prime}:=\left\{l \in N(i) \text { : there is a } p=\left(k_{1}, \ldots, k_{r}\right) \in D_{x} \text { with } l=k_{2}\right\} .
$$

As in Example 2.1 it takes the server a random time $W^{1}$ with some distribution $F_{i j}^{\prime}$ to walk from $i$ to $j$. If $X_{j}\left(W^{1}\right)>0$, then we put $W_{1}:=W^{1}$. If not, then the server chooses another station randomly in the set $D_{X\left(W_{1}\right)}^{\prime}$. The server continues in this way until he arrives at a nonempty station at a time $W_{1}$. If $\sum_{k} x_{k}=0$, then the server moves as in the previous example. Once the server has completed a walk at a moment where the system is nonempty, he continues walking as described above. It is easy to see that (2.3) and (2.4) hold. This example could be generalized by allowing the server to change his destination during a walk if there occurs an arrival at a station that is closer than that he is currently walking to. In this case the choice of the next destination depends again on future arrivals.

State-independent routings as in Fricker and Jaibi (1994) are also covered by our model. A special case is a Markovian routing as described next.

EXAMPLE 2.3. Let $\left(r_{i, j}\right)_{1 \leq i, j \leq K}$ be an irreducible Markovian (routing) matrix and assume that the server, upon completing a batch of services at station $i$, chooses his next destination $j$, say, with probability $r_{i, j}$ independent of everything else. It takes the server a random time with distribution $F_{i j}^{\prime}$ to walk from $i$ to $j$. These walking times are independent of each other and independent of anything else. If at the moment of the server's arrival the $j$ th queue is nonempty, then it is served according to the exhaustive policy. Otherwise the server chooses a next destination according to the routing matrix. Assuming that the $F_{i j}^{\prime}$ have finite means $w_{i j}$ satisfying $\sum_{i j} w_{i j}>0$, both conditions (2.3) and (2.4) are fulfilled. 
We conclude with a simple example where the only nonzero walking times are times taken to wait for the next arriving customer. Such a system is very similar to an M/GI/1-queueing system with batch arrivals. In particular, it is rather easy to see that stability is implied by inequality (1.1).

EXAMPLE 2.4. Assume that after a batch of services the system is not empty. Then the server chooses one of the nonempty stations and resumes servicing instantaneously. If, on the other hand, the system is empty after a batch of services, then the server waits for the next batch of arriving customers. Then he chooses one of the nonempty stations and starts serving without further delay.

3. Proof of the stability result. We consider the Markov chain $\{\hat{X}(n)\}$ defined by (2.5). All assumptions formulated in the previous section are supposed to be in force. The main result of our paper is the following theorem.

THEOREM 3.1. (i) Assume that (1.1) holds. Then there is a finite subset of $\mathbb{Z}_{+}^{K} \times\{1, \ldots, K\}$ which is positive recurrent for the Markov chain $\{\hat{X}(n)\}$. If the chain is irreducible, then it is positive recurrent and if it is, in addition, also aperiodic, then it is ergodic.

(ii) If the chain $\{\hat{X}(n)\}$ is ergodic, then (1.1) holds.

REMARK 3.2. In Examples 2.1 through 2.4 the Markov chain $\{\hat{X}(n)\}$ is irreducible and aperiodic. In general, these properties are not implied by our model assumptions and must be checked in each case.

The proof of the theorem is split into several lemmas. The basic idea is to prove that the number of walks can be neglected when compared with the number of services. A polling system where the server needs no time to travel to a nonempty station as in Example 2.4 can easily be proved to be stable if (1.1) is satisfied.

Unless stated otherwise we consider in this section a more general model, where the stochastic behavior of the system is influenced by a further piecewise constant process $\{U(t): t \geq 0\}$ taking values in some measurable space $(\mathbf{U}, \mathscr{U})$ and being right continuous w.r.t. the discrete topology. We let

$$
X(t):=\left(X_{1}(t), \ldots, X_{K}(t), S(t), U(t)\right)
$$

and define the filtration $\left\{\mathscr{F}_{t}\right\}$ as before, where we now use the new process $X(t)$. We apply the assumptions on the arrival process and the services of Section 2 verbatim and we also assume that

$$
\hat{X}(n):=\left(X_{1}\left(T_{n}\right), \ldots, X_{K}\left(T_{n}\right), \hat{S}(n), U\left(T_{n}\right)\right)
$$

is a homogeneous Markov process. We can assume that it fits the general setting of the Appendix. Our assumptions (2.3) and (2.4) remain unchanged, but we note that $\mathscr{F}_{0}$ also contains the information induced by $U(0)$. 
Define $\nu_{0}:=0$ and

$$
\nu_{n+1}:=\min \left\{k>\nu_{n}: \hat{X}_{\hat{S}(k)}(k)=0\right\}, \quad n \in \mathbb{Z}_{+} .
$$

If $\hat{X}_{\hat{S}(0)}=0$ (resp., $>0$ ), then $\nu_{n}, n \in \mathbb{N}$, is the number of completed services before the server starts his $(n+1)$ th (resp. $n$ th) walk. Clearly, $T_{\nu_{n}}$ is an $\left\{\mathscr{F}_{t}\right\}$-stopping time. Most of our analysis will be based on the Markov chain $\left(Y(n), \mathscr{G}_{n}\right), n \in \mathbb{Z}_{+}$, which is defined by

$$
Y(n)=:\left(Y_{1}(n), \ldots, Y_{K}(n), S_{n}, U_{n}\right):=\hat{X}\left(\nu_{n}\right)=X\left(T_{\nu_{n}}\right), \quad \mathscr{G}_{n}:=\mathscr{F}_{T_{\nu_{n}}} .
$$

Note that $\left(Y(0), \mathscr{G}_{0}\right)=\left(X(0), \mathscr{F}_{0}\right)$.

We define a function $V$ on $\mathbb{Z}_{+}^{K} \times\{1, \ldots, K\} \times \mathbf{U}$ by

$$
V(x):=b_{1} x_{1}+\cdots+b_{K} x_{K}, \quad x=\left(x_{1}, \ldots, x_{K}, i, u\right) .
$$

Further we define $|x|:=x_{1}+\cdots+x_{K}$, where $x$ is as above. that

Lemma 3.3. Assume (1.1). There are constants $d_{1}, d_{2}, d_{1}^{\prime}, d_{2}^{\prime}, \tilde{d}_{1}, \tilde{d}_{2}$ such

PRoOF. Unless stated otherwise we assume that $\hat{X}_{\hat{S}(0)}(0)=0$.

At time $T^{1}$ the server enters station $\hat{S}(1)$ and then the system operates [independently of $U(0)$ ] like a stable M/GI/1-queueing system (with batch arrivals) until station $\hat{S}(1)$ is empty. It is well known that the busy period $T_{\nu_{1}}-T^{1}$ satisfies

$$
E\left[T_{\nu_{1}}-T^{1} \mid \mathscr{F}_{T^{1}}\right] \leq h\left(X_{S\left(T^{1}\right)}\left(T^{1}\right)\right), \quad P \text {-a.s., }
$$

where $h$ is a linear function and where the independence assumptions have also been used. Indeed, assume that $S\left(T^{1}\right)=i$ and consider a random walk generated by the increment $A_{i}\left(\eta_{i}\right)-1$, where $\eta_{i}$ is a generic service time at station $i$ independent of $A_{i}$. Then

$$
E\left[\nu_{1} \mid \mathscr{F}_{T^{1}}\right]=c_{i} Y_{i}\left(T^{1}\right),
$$

where $c_{i}$ is the mean of the number of steps needed by the random walk to decrease by 1 . Now the desired inequality follows from Wald's identity. Using our assumption (2.3), we hence obtain that

From (2.1) we have

$$
\begin{aligned}
E\left[T_{\nu_{1}} \mid \mathscr{G}_{0}\right] & =E\left[W_{1} \mid \mathscr{G}_{0}\right]+E\left[E\left[T_{\nu_{1}}-T^{1} \mid \mathscr{F}_{T^{1}}\right] \mid \mathscr{G}_{0}\right] \\
& \leq w+E\left[h\left(X_{S\left(T^{1}\right)}\left(T^{1}\right)\right) \mid \mathscr{G}_{0}\right] .
\end{aligned}
$$

$$
\begin{aligned}
E\left[X_{S\left(T^{1}\right)}\left(T^{1}\right) \mid \mathscr{G}_{0}\right] & \leq|Y(0)|+\sum_{i} E\left[A_{i}\left(T^{1}\right) \mid \mathscr{G}_{0}\right] \\
& \leq|Y(0)|+w \sum_{i} \lambda_{i}
\end{aligned}
$$


and (3.3) follows. Similarly,

$$
\begin{aligned}
E\left[Y_{i}(1) \mid \mathscr{G}_{0}\right] & \leq Y_{i}(0)+E\left[A_{i}\left(T_{\nu_{1}}\right) \mid \mathscr{G}_{0}\right] \\
& \leq Y_{i}(0)+\lambda_{i} E\left[T_{\nu_{1}} \mid \mathscr{G}_{0}\right]
\end{aligned}
$$

and (3.4) follows. The third assertion follows by similar arguments.

If $\hat{X}_{\hat{S}(0)}(0)>0$, then the assertions follow by directly referring to the properties of an M/GI/1-system.

LEMma 3.4. Consider a measurable set $B_{\infty} \subseteq \mathbb{Z}_{+}^{K} \times\{1, \ldots, K\} \times \mathbf{U}$ and assume that there is a $C \in \mathscr{F}_{0}$ satisfying

$$
P\left(T_{\infty}<\tau_{1} \mid \mathscr{F}_{T_{n}}\right)>p \quad \text { P-a.s. on }\left\{\left|X\left(T_{n}\right)\right|=0\right\} \cap C, n \in \mathbb{Z}_{+},
$$

where

$$
T_{\infty}:=\inf \left\{t: X(t) \in B_{\infty}\right\}
$$

and where we recall that $\tau_{1}$ is the first arrival epoch. Then, for any number $N>0$, there is a $p(N)>0$ satisfying

$$
P\left(T_{\infty}<\tau_{1} \mid \mathscr{F}_{0}\right) \geq p(N), \quad P \text {-a.s. on }\{|X(0)| \leq N\} .
$$

Proof. Because we could always argue on the set $C$, we will assume for simplicity that $C=\Omega$. Given $\mathscr{F}_{0}$, one can determine the number $\xi$ of nonempty stations. Assume for simplicity that $X_{S_{0}}(0)>0$ and put

$$
T:=\sum_{i=1}^{\nu_{1}}\left(T_{i}-T^{i}\right)+W_{\nu_{1}+1}+\cdots+W_{\nu_{\xi}-1}+\sum_{i=\nu_{\xi-1}+1}^{\nu_{\xi}}\left(T_{i}-T^{i}\right) .
$$

Then $\left\{A(T)=0, A\left(T_{\infty}\right)=0, X\left(T^{\nu_{\xi}+1}\right) \in B_{\infty}\right\} \subseteq\left\{T_{\infty}<\tau_{1}\right\}$ and we may use our assumptions (2.4), (3.6) and successive conditioning to obtain the estimate

where

$$
P\left(T_{\infty}<\tau_{1} \mid \mathscr{F}_{0}\right) \geq \tilde{p}^{|X(0)|} p^{\xi+1},
$$

$$
\tilde{p}:=\min _{j} P\left(A\left(T_{1}-T^{1}\right)=0 \mid S\left(T^{1}\right)=j\right)>0 .
$$

For $|X(0)| \leq N$ the right-hand side of the above inequality is not smaller than $p(N):=\tilde{p}^{N} p^{K+1}$.

We need to introduce some further notation. Let $\left\{\theta_{n}: n \in \mathbb{Z}_{+}\right\}$be the flow of shift operators associated with the process $\{\hat{X}(n)\}$; see the Appendix. Then

$$
\theta^{\prime}:=\theta_{\nu_{1}}
$$

is the shift operator associated with $\{Y(n)\}$. The symbol $O$ is reserved to denote a (deterministic) function $O: \mathbb{R}_{+} \rightarrow \mathbb{R}_{+}$satisfying lim $\sup _{t \rightarrow \infty} O(t) / t<$ $\infty$. Further we let

$$
D(t):=\operatorname{card}\left\{n \geq 1: T_{n} \leq t\right\}
$$

be the number of departures by time $t$.

Under (1.1) the assumptions of the following lemma will be proved to hold. 
LEMMA 3.5. Assume that $\sigma$ is a $\left\{\mathscr{G}_{n}\right\}$-stopping time and $L, c_{1}, c_{2} \in \mathbb{R}_{+}$, $\varepsilon, c \in(0,1)$ are constants satisfying

$$
\begin{aligned}
E\left[V(Y(\sigma)) \mid \mathscr{G}_{0}\right] & \leq c V(Y(0)), & & \text { P-a.s. on }\{V(Y(0))>L\}, \\
E\left[\sigma \mid \mathscr{G}_{0}\right] & \leq c_{1} V(Y(0))^{1-\varepsilon}, & & \text { P-a.s. on }\{V(Y(0))>L\}, \\
E\left[\nu_{\sigma} \mid \mathscr{G}_{0}\right] & \leq c_{2} V(Y(0)), & & \text { P-a.s. on }\{V(Y(0))>L\} .
\end{aligned}
$$

Then, under the assumptions of Lemma 3.4,

$$
\begin{aligned}
E\left[\tau_{\infty} \mid \mathscr{G}_{0}\right] & =O\left(V(Y(0))^{1-\varepsilon}\right), & & P \text {-a.s. on } C, \\
E\left[D\left(T_{\infty}\right) \mid \mathscr{G}_{0}\right] & =O(V(Y(0))), & & \text { P-a.s. on } C,
\end{aligned}
$$

where $T_{\infty}$ is given by (3.7),

$$
\tau_{\infty}:=\min \left\{n \geq 1: Y(n) \in B_{\infty}\right\}
$$

and $\min \varnothing:=\infty$.

Proof. Define $\sigma_{0}:=0$ and

$$
\begin{aligned}
\sigma_{n+1} & :=\sigma_{n}+\sigma \circ \theta_{\sigma_{n}}^{\prime}, \quad n \in \mathbb{Z}_{+}, \\
\gamma & :=\min \left\{n \geq 1: V\left(Y\left(\sigma_{n}\right)\right) \leq c V(Y(0))\right\},
\end{aligned}
$$

where $\theta_{0}^{\prime}$ denotes the identity on $\Omega$. By (3.8) and Lemma 4.2 we have

$$
E\left[\gamma \mid \mathscr{G}_{0}\right] \leq \frac{1}{(1-c) c}, \quad P \text {-a.s. on }\left\{V(Y(0))>L^{\prime}\right\},
$$

where $L^{\prime}:=L / c$. The $\left\{\mathscr{G}_{n}\right\}$-stopping time $\sigma^{\prime}:=\sigma_{\gamma}$ satisfies

$$
V\left(Y\left(\sigma^{\prime}\right)\right) \leq c V(Y(0))
$$

everywhere on $\{\gamma<\infty\}$. Moreover, by (3.10) we have, $P$-almost surely on $\left\{V(Y(0))>L^{\prime}\right\}$, that

$$
\begin{aligned}
E\left[\nu_{\sigma^{\prime}} \mid \mathscr{G}_{0}\right] & =E\left[\sum_{j=0}^{\gamma-1}\left(\nu_{\sigma_{j+1}}-\nu_{\sigma_{j}}\right) \mid \mathscr{G}_{0}\right] \\
& =E\left[\sum_{j=0}^{\infty} \mathbf{1}\{j<\gamma\} E\left[\left(\nu_{\sigma_{j+1}}-\nu_{\sigma_{j}}\right) \mid \mathscr{G}_{\sigma_{j}}\right] \mid \mathscr{G}_{0}\right] \\
& \leq c_{2} E\left[\sum_{j=0}^{\infty} \mathbf{1}\{j<\gamma\} V\left(Y\left(\sigma_{j}\right)\right) \mid \mathscr{G}_{0}\right] \\
& \leq c_{2} E\left[\sum_{j=0}^{\gamma} V\left(Y\left(\sigma_{j}\right)\right) \mid \mathscr{G}_{0}\right] .
\end{aligned}
$$


From Lemma 4.2 we obtain that

$$
E\left[\nu_{\sigma^{\prime}} \mid \mathscr{G}_{0}\right] \leq c_{2}^{\prime} V(Y(0)), \quad P \text {-a.s. on }\left\{V(Y(0))>L^{\prime}\right\},
$$

where $c_{2}^{\prime}:=c_{2} /(1-c)$. In a similar way we obtain from (3.9) that

$$
E\left[\sigma^{\prime} \mid \mathscr{G}_{0}\right]=E\left[\sum_{j=0}^{\gamma-1} \sigma \circ \theta_{\sigma_{j}}^{\prime} \mid \mathscr{G}_{0}\right] \leq c_{1} E\left[\sum_{j=0}^{\gamma} V\left(Y\left(\sigma_{j}\right)\right)^{1-\varepsilon} \mid \mathscr{G}_{0}\right],
$$

so that, by Lemma 4.2,

$$
E\left[\sigma^{\prime} \mid \mathscr{G}_{0}\right] \leq c_{1}^{\prime} V(Y(0))^{1-\varepsilon}, \quad P \text {-a.s. on }\left\{V(Y(0))>L^{\prime}\right\},
$$

where $c_{1}^{\prime}:=c_{1} /(1-c)$. Next we iterate $\sigma^{\prime}$ by defining $\sigma_{0}^{\prime}:=0$ and

$$
\sigma_{n+1}^{\prime}:=\sigma_{n}^{\prime}+\sigma^{\prime} \circ \theta_{\sigma_{n}^{\prime}}^{\prime}, \quad n \in \mathbb{Z}_{+} .
$$

Since $Y\left(\sigma_{n+1}^{\prime}\right)=Y\left(\sigma^{\prime}\right) \circ \theta_{\sigma_{n}^{\prime}}^{\prime}$, we have

$$
V\left(Y\left(\sigma_{n}^{\prime}\right)\right) \leq c^{n} V(Y(0)) \text {. }
$$

Denote

$$
\tau^{\prime}:=\min \left\{n \geq 1: V(Y(n)) \leq L^{\prime}\right\} .
$$

Then we can bound $\tau^{\prime} \leq \sigma_{\gamma^{\prime}}^{\prime}$, where

$$
\gamma^{\prime}:=\min \left\{n \geq 1: V\left(Y\left(\sigma_{n}^{\prime}\right)\right) \leq L^{\prime}\right\} .
$$

Arguing as above, we get from (3.15) and (3.16), $P$-almost surely on $\left\{V(Y(0))>L^{\prime}\right\}$, that

$$
\begin{aligned}
E\left[\tau^{\prime} \mid \mathscr{G}_{0}\right] & \leq E\left[\sum_{j=0}^{\gamma^{\prime}-1}\left(\sigma_{j+1}^{\prime}-\sigma_{j}^{\prime}\right) \mid \mathscr{G}_{0}\right] \\
& =\sum_{j=0}^{\infty} E\left[\mathbf{1}\left\{j<\gamma^{\prime}\right\} E\left[\sigma^{\prime} \circ \theta_{\sigma_{j}^{\prime}}^{\prime} \mid \mathscr{G}_{\sigma_{j}^{\prime}}\right] \mid \mathscr{G}_{0}\right] \\
& \leq c_{1}^{\prime} \sum_{j=0}^{\infty} E\left[\mathbf{1}\left\{j<\gamma^{\prime}\right\} V\left(Y\left(\sigma_{j}^{\prime}\right)\right)^{1-\varepsilon} \mid \mathscr{G}_{0}\right] \\
& \leq c_{1}^{\prime} V(Y(0))^{1-\varepsilon} \sum_{j=0}^{\infty} c^{(1-\varepsilon) j}
\end{aligned}
$$

and hence

$$
E\left[\tau^{\prime} \mid \mathscr{G}_{0}\right] \leq \tilde{c}_{1} V(Y(0))^{1-\varepsilon}, \quad P \text {-a.s. on }\left\{V(Y(0))>L^{\prime}\right\},
$$

where $\tilde{c}_{1}:=c_{1}^{\prime} /\left(1-c^{(1-\varepsilon)}\right)$. Similarly, we obtain from (3.14) that

$$
E\left[\nu_{\tau^{\prime}} \mid \mathscr{G}_{0}\right] \leq \tilde{c}_{2} V(Y(0)), \quad P \text {-a.s. on }\left\{V(Y(0))>L^{\prime}\right\},
$$

where $\tilde{c}_{2}:=c_{2}^{\prime} /(1-c)$.

To prove (3.11) and (3.12), we define inductively, for $\in \mathbb{Z}_{+}$,

$$
\begin{aligned}
& T_{n+1}^{\prime}:=\inf \left\{t \geq T_{n}^{*}: V(X(t)) \leq L^{\prime}\right\}, \\
& T_{n+1}^{*}:=\inf \left\{t>T_{n+1}^{\prime}: A(t)>A(t-)\right\},
\end{aligned}
$$


where $T_{0}^{*}:=0$. Note that $T_{n+1}^{\prime}=T_{n}^{*}$ if $V\left(X\left(T_{n}^{*}\right)\right) \leq L^{\prime}$. Let

$$
B(t):=\operatorname{card}\left\{n \geq 1: T_{\nu_{n}} \leq t\right\}
$$

be the number of completed batches of services by time $t$. We have the following inequalities:

$$
T_{1}^{\prime} \leq T_{\nu_{r^{\prime}}}, \quad D\left(T_{1}^{\prime}\right) \leq \nu_{\tau^{\prime}}, \quad B\left(T_{1}^{\prime}\right) \leq \tau^{\prime} .
$$

Let

$$
\sigma^{*}:=\min \left\{n \geq 1: X(t) \in B_{\infty} \text { for some } t \text { with } T_{n}^{\prime} \leq t \leq T_{n}^{*}\right\} .
$$

On the set $\left\{V(X(0))>L^{\prime}\right\}$ we have, by (3.18) and (3.19),

$$
\begin{aligned}
E\left[D\left(T_{\sigma^{*}}^{*}\right) \mid \mathscr{F}_{0}\right] & \\
= & E\left[\sum_{j=0}^{\infty} \mathbf{1}\left\{j<\sigma^{*}\right\}\left(D\left(T_{j+1}^{*}\right)-D\left(T_{j}^{*}\right)\right) \mid \mathscr{F}_{0}\right] \\
= & E\left[\sum_{j=0}^{\infty} \mathbf{1}\left\{j<\sigma^{*}\right\}\left(D\left(T_{j+1}^{*}\right)-D\left(T_{j+1}^{\prime}\right)\right) \mid \mathscr{F}_{0}\right] \\
& +E\left[\sum_{j=0}^{\infty} \mathbf{1}\left\{j<\sigma^{*}\right\} E\left[D\left(T_{j+1}^{\prime}\right)-D\left(T_{j}^{*}\right) \mid \mathscr{F}_{T_{j}^{*}}\right] \mid \mathscr{F}_{0}\right] \\
& L^{\prime \prime} E\left[\sigma^{*} \mid \mathscr{F}_{0}\right]+\tilde{c}_{2} E\left[\sum_{j=0}^{\infty} \mathbf{1}\left\{j<\sigma^{*}\right\} \mathbf{1}\left\{V\left(X\left(T_{j}^{*}\right)\right)>L^{\prime}\right\} V\left(X\left(T_{j}^{*}\right)\right) \mid \mathscr{F}_{0}\right] \\
\leq & \left(L^{\prime \prime}+\tilde{c}_{2}\left(L^{\prime}+1\right)\right) E\left[\sigma^{*} \mid \mathscr{F}_{0}\right]+\tilde{c}_{2} V(X(0)),
\end{aligned}
$$

where $L^{\prime \prime}$ is a finite constant satisfying $|x| \leq L^{\prime \prime}$ whenever $V(x) \leq L^{\prime}$. Here we have also used, for $n \geq 1$, the easily proved inequality

$$
E\left[V\left(X\left(T_{n}^{*}\right)\right) \mid \mathscr{F}_{T_{n}^{\prime}}\right] \leq L^{\prime}+\max _{i} \lambda_{i} b_{i},
$$

where we assume for notational simplicity that $\lambda_{i} b_{i} \leq 1$ for all $i$. Similarly, we obtain from (3.17) and (3.19) that

$$
E\left[B\left(T_{\sigma^{*}}^{*}\right) \mid \mathscr{F}_{0}\right] \leq\left(K+\tilde{c}_{1}\left(L^{\prime}+1\right)^{1-\varepsilon}\right) E\left[\sigma^{*} \mid \mathscr{F}_{0}\right]+\tilde{c}_{2} V(X(0))^{1-\varepsilon} .
$$

By standard arguments it follows that $\tau^{\prime \prime}:=B\left(T_{\sigma^{*}}^{*}\right)$ is a $\left\{\mathscr{G}_{n}\right\}$-stopping time. Since clearly $\nu_{\tau^{\prime \prime}} \leq D\left(T_{\sigma^{*}}^{*}\right)$ and $\tau_{\infty} \leq \tau^{\prime \prime}$, we could use our estimates to conclude the assertions (3.11) and (3.12) if we knew that $E\left[\sigma^{*} \mid \mathscr{F}_{0}\right]$ is bounded on $\left\{V(X(0))>L^{\prime}\right\}$. To this end we note that Lemma 3.4 implies, $P$-a.s. on $C$,

$$
\left.P\left(X(t) \in B_{\infty} \text { for some } t \text { with } T_{n}^{\prime} \leq t \leq T_{n}^{*}\right\} \mid \mathscr{F}_{T_{n}^{\prime}}\right) \geq p\left(L^{\prime \prime}\right),
$$

so that a geometrical trial argument completes the proof.

Lemma 3.6. Assume (1.1). Then there exists $a\left\{\mathscr{G}_{n}\right\}$-stopping time $\sigma$ and constants $L, c_{1}, c_{2} \in \mathbb{R}_{+}, \varepsilon, c \in(0,1)$, such that (3.8)-(3.10) in Lemma 3.5 are satisfied. 
Proof. We proceed by induction on the number of stations. For $K=1$ we can take $\sigma=1$. Then (3.8) and (3.9) are trivial, while (3.10) is a well-known property of a stable M/GI/1-queueing system; see Lemma 3.3.

Now we suppose that the assertion is true for all polling systems with $K$ stations satisfying our general assumptions and (1.1). We consider a polling system with $K+1$ stations described by the process

$$
X(t)=\left(X_{1}(t), \ldots, X_{K+1}(t), S(t), U(t)\right) .
$$

To exploit our induction hypothesis, we couple $X(t)$ with another auxiliary polling system with stations $\{1, \ldots, K\}$ described by the process $\tilde{X}(t)$. This auxiliary process should behave like the original process until the time when the server first enters station $K+1$. Hence we define

$$
\left(\tilde{X}_{1}(t), \ldots, \tilde{X}_{K}(t), \tilde{S}(t)\right):=\left(X_{1}(t), \ldots, X_{K}(t), S(t)\right), \quad t<\tilde{T}_{\infty},
$$

where

$$
\tilde{T}_{\infty}:=\inf \{t: S(t)=K+1\} .
$$

Further we let $\tilde{U}(t):=\left(U(t), X_{K+1}(0)+A_{k+1}(t)\right)$ for $t<\tilde{T}_{\infty}$ and $\tilde{U}(t)=u_{\infty}$ for $t \geq \tilde{T}_{\infty}$, where $u_{\infty}$ is not in the space $\mathbf{U} \times \mathbb{Z}_{+}$. Hence the process $\tilde{U}(t)$ takes values in the set $\mathbf{U} \times \mathbb{Z}_{+} \cup\left\{u_{\infty}\right\}$. Assume that $\tilde{T}_{\infty}=T^{n+1}$ for $n \in \mathbb{Z}_{+}$. In particular, $X_{S\left(T_{n}-\right)}\left(T_{n}\right)=0$ and we recall that $T^{n+1}=T_{n}$ is possible. We distinguish two cases. First we assume that $\sum_{i \neq K+1} X_{i}\left(T_{n}\right)>0$. Then we let $\tilde{T}^{n+1}:=T^{n+1}$ be the moment of the beginning of the $(n+1)$ th service for the process $\{\tilde{X}(t)\}$ and assume that

$$
\tilde{X}\left(T^{n+1}\right)=\left(X_{1}\left(T^{n+1}\right), \ldots, X_{K}\left(T^{n+1}\right), \tilde{S}\left(T^{n+1}\right), u_{\infty}\right),
$$

where $\tilde{S}\left(T^{n+1}\right)$ is assumed to be in the set $\left\{j \neq K+1: X_{j}\left(T^{n+1}\right)>0\right\}$. The second case is $\sum_{i \neq K+1} X_{i}\left(T^{n+1}\right)=0$. In that case we put

$$
\tilde{T}^{n+1}:=\inf \left\{t>T^{n+1}: \sum_{i \neq K+1} A_{i}(t)>\sum_{i \neq K+1} A(t-)\right\}
$$

and

$$
\tilde{X}_{i}(t)=X_{i}\left(T_{n}\right)+A_{i}\left(T_{n}, t\right], \quad T_{n} \leq t \leq \tilde{T}^{n+1} .
$$

Further we set $S(t)=0$ for $T_{n} \leq t<\tilde{T}^{n+1}$ and we assume that $\tilde{S}\left(T^{n+1}\right)$ is an element of $\left\{i \neq K+1: \tilde{X}_{i}\left(\tilde{T}^{n+1}\right)>0\right\}$. In both cases we assume that the process $\left(\tilde{X}_{1}(t), \ldots, \tilde{X}_{K}(t), \tilde{S}(t)\right)$ evolves after time $\tilde{T}^{n+1}$ as in Example 2.4.

So far we have given a pathwise description of the process $\tilde{X}(t)$. We skip here the obvious technical details describing the joint distribution of $\{\tilde{X}(t)\}$ and $\{X(t)\}$ and ensuring that $\{\tilde{X}(t)\}$ will indeed become a process satisfying the general assumptions of this section. Assumption (2.4) also implies (3.6) for $B_{\infty}:=\mathbb{Z}_{+}^{K} \times\{1, \ldots, K\} \times\left\{u_{\infty}\right\}$ and $C=\left\{X_{K+1}(0)>0\right\}$, a set which is $\sigma\{\tilde{X}(0)\}$ measurable by definition. Hence we can apply the induction hypothesis to obtain (3.11) and (3.12) with $\tau_{\infty}$ replaced by

$$
\tilde{\tau}_{\infty}:=\min \left\{n \geq 1: S_{n}=K+1\right\}
$$


and $T_{\infty}$ replaced by $\tilde{T}_{\infty}$. This argument works just as well for any station other than $K+1$.

There is a $\mathscr{G}_{0}$-measurable random element $\xi$ of $\{1, \ldots, K+1\}$ with the property

$$
Y_{\xi}(0) \geq \frac{|Y(0)|}{K+1} .
$$

We claim that (3.8)-(3.10) are satisfied with

$$
\sigma:= \begin{cases}\min \left\{n \geq 1: \xi=S_{n}\right\}, & \text { if } S_{0} \neq \xi \\ 0, & \text { otherwise. }\end{cases}
$$

Since $\xi$ is $\mathscr{G}_{0}$-measurable we can use the above argument to conclude that

$$
\begin{aligned}
E\left[\sigma \mid \mathscr{G}_{0}\right] & =O\left(V(Y(0))^{1-\varepsilon}\right), \\
E\left[D(\tilde{T}) \mid \mathscr{G}_{0}\right] & =O(V(Y(0))),
\end{aligned}
$$

where

$$
\tilde{T}:=\inf \{t \geq 0: S(t)=\xi\} .
$$

Next we are going to show that $\sigma$ does satisfy the drift condition (3.8). We have

$$
Y_{i}(\sigma)=Y_{i}(0)+A_{i}\left(T_{\nu_{\sigma}}\right)-\nu_{\sigma}^{i},
$$

where

$$
\nu_{n}^{i}:=\operatorname{card}\left\{k \in \mathbb{N}: k \leq \nu_{n}: \hat{S}(k)=i\right\}, \quad n \in \mathbb{N} .
$$

Using the very definitions of the polling system as well as (2.2) and (2.3), we can calculate

$$
\begin{aligned}
E\left[T_{\nu_{\sigma}} \mid \mathscr{F}_{0}\right]= & E\left[\sum_{m=0}^{\infty} \mathbf{1}\{m+1 \leq \sigma\}\left(W_{\nu_{m}+1}+\sum_{n=\nu_{m}+1}^{\nu_{m}+1}\left(T_{n}-T^{n}\right)\right) \mid \mathscr{F}_{0}\right] \\
= & E\left[\sum_{m=0}^{\infty} \mathbf{1}\{m+1 \leq \sigma\} E\left[W_{\nu_{m}+1} \mid \mathscr{F}_{T_{\nu_{m}}}\right] \mid \mathscr{F}_{0}\right] \\
& +E\left[\sum_{m=0}^{\infty} \mathbf{1}\{m+1 \leq \sigma\} \sum_{i=1}^{K+1} \mathbf{1}\left\{S\left(T^{\nu_{m}+1}\right)=i\right\}\right. \\
\leq & \left.\times \sum_{n=1}^{\infty} \mathbf{1}\left\{\nu_{m}+1 \leq n \leq \nu_{m+1}\right\} E\left[T_{n}-T^{n} \mid \mathscr{F}_{T^{n}}\right] \mid \mathscr{F}_{0}\right] \\
& +E\left[\sum_{m=0}^{\infty} \mathbf{1}\{m+1 \leq \sigma\} \mid \mathscr{F}_{0}\right] \\
& \left.\quad \sum_{m=0}^{\infty} \mathbf{1}\{m+1 \leq \sigma\} \sum_{i=1}^{K+1} \mathbf{1}\left\{S\left(T^{\nu_{m}+1}\right)=i\right\} b_{i}\left(\nu_{m+1}-\nu_{m}\right) \mid \mathscr{F}_{0}\right]
\end{aligned}
$$


where we have taken advantage of the stopping time properties of $\sigma$ and $\nu_{m}$ which imply, for example, that

$$
\left\{m+1 \leq \sigma, S\left(T^{\nu_{m}+1}\right)=i, \nu_{m}+1 \leq n \leq \nu_{m+1}\right\} \in \mathscr{F}_{T^{n}} .
$$

Hence

$$
E\left[T_{\nu_{\sigma}} \mid \mathscr{F}_{0}\right] \leq w E\left[\sigma \mid \mathscr{F}_{0}\right]+E\left[\zeta \mid \mathscr{F}_{0}\right]
$$

where

$$
\zeta:=\sum_{i=1}^{K+1} b_{i} \nu_{\sigma}^{i}
$$

Using

$$
E\left[A_{i}\left(T_{\nu_{\sigma}}\right) \mid \mathscr{F}_{0}\right]=\lambda_{i} E\left[T_{\nu_{\sigma}} \mid \mathscr{F}_{0}\right],
$$

we obtain from (3.22) and (3.24) that

$$
\begin{gathered}
E\left[V(Y(\sigma)) \mid \mathscr{F}_{0}\right] \leq \\
V(Y(0))-(1-\rho) E\left[\zeta \mid \mathscr{F}_{0}\right] \\
+O\left(V(Y(0))^{1-\tilde{\varepsilon}}\right),
\end{gathered}
$$

where the workload

$$
\rho:=\sum_{i=1}^{K+1} \lambda_{i} b_{i}
$$

is strictly less than 1 by assumption. From the definitions (3.20) and (3.21) we have

$$
\nu_{\sigma}=\sum_{i=1}^{K+1} \nu_{\sigma}^{i} \geq \frac{|Y(0)|}{K+1}
$$

yielding (since all $b_{i}$ are positive) that

$$
E\left[\zeta \mid \mathscr{F}_{0}\right] \geq \tilde{c} V(Y(0)),
$$

where

$$
\tilde{c}=\frac{\min b_{i}}{(K+1) \max b_{i}} .
$$

Inserting this into (3.26), we obtain (3.8) for a suitably chosen constant $L$.

Next we want to prove (3.10) making use of the equality

$$
E\left[\nu_{\sigma} \mid \mathscr{F}_{0}\right]=E\left[D(\tilde{T}) \mid \mathscr{F}_{0}\right]+E\left[E\left[D\left(T_{\nu_{\sigma}}\right)-D(\tilde{T}) \mid \mathscr{F}_{\tilde{T}}\right] \mid \mathscr{F}_{0}\right] .
$$

The first summand can be bounded according to (3.23). For the second we can use (3.5) and

$$
E\left[V(X(\tilde{T})) \mid \mathscr{F}_{0}\right]=O(V(X(0))),
$$

which can be proved as (3.26).

In the remainder of this section we return to the setting of Section 2 which is obtained by letting $U(t) \equiv 0$. 
Proof of Theorem 3.1(i). Assume (1.1). By Lemma 3.6 we can use Theorem 4.3 with $\left(\hat{X}(n), \nu_{\sigma}\right)$ in place of $(X(n), \sigma)$. Assumption (4.6) of that theorem is clearly satisfied and the set $\{x: V(x) \leq L\}$ is finite for any $L>0$. The other assertions are standard.

In case $\{Y(n)\}$ is ergodic we let $\pi$ denote its stationary initial distribution and define

$$
\bar{w}:=\sum_{x} w(x) \pi(x),
$$

where $w(x), x \in \mathbb{Z}_{+}^{K} \times\{1, \ldots, K\}$, satisfies

$$
w(x)=E\left[W_{1} \mid \mathscr{F}_{0}\right], \quad P \text {-a.s. on }\{Y(0)=x\} .
$$

Hence $\bar{w}$ is the average time taken by a walk after a batch of services. Since $\pi((0, \ldots, 0, i))>0$ for all $i \in\{1, \ldots, K\}$, it is clear from the definition of $W_{1}$ that $\bar{w}$ has to be positive. Consider now the Markov chain $Y^{\prime}(n)$ denoting the system states at the consecutive polling instants, that is,

$$
Y^{\prime}(n):=X\left(T^{\nu_{n}+1}\right), \quad n \in \mathbb{Z}_{+},
$$

and let $\pi^{\prime}$ denote its equilibrium distribution if the chain is ergodic. Note that $\pi^{\prime}$ has to be concentrated on the set of all those $x=\left(x_{1}, \ldots, x_{K}, i\right)$ satisfying $x_{i}>0$. On this set we define a function $B(\cdot)$ by

$$
B(x)=E\left[\nu_{1} \mid \mathscr{F}_{0}\right] \quad P \text {-a.s. on }\{Y(0)=x\} .
$$

Let

Then

$$
\pi_{i}:=\pi^{\prime}\left(\mathbb{Z}_{+}^{K} \times\{i\}\right)=\pi\left(\mathbb{Z}_{+}^{K} \times\{i\}\right) .
$$

$$
\bar{B}_{i}:=\pi_{i}^{-1} \sum_{x \in \mathbb{Z}_{+}^{K}} B((x, i)) \pi^{\prime}(\{(x, i)\})
$$

is the stationary average batch size given that the server is at station $i$.

Next we turn to the proof of the necessity part of Theorem 3.1. This proof will also yield an equilibrium equation for the process $\{\hat{X}(n)\}$. A shorter proof could be obtained by comparing the system with a polling system as in Example 2.4. The latter system can easily be proved to be unstable if (1.1) does not hold.

Proof of NECESSity of (1.1). Let $n \in \mathbb{N}$. We can use the calculations which led to (3.25) (with $n$ in place of $\sigma$ ) to obtain that

$$
E\left[Y_{i}(n) \mid \mathscr{F}_{0}\right]=Y_{i}(0)+\lambda_{i} E\left[\left(W(n)+\zeta_{n}\right) \mid \mathscr{F}_{0}\right]-E\left[\nu_{n}^{i} \mid \mathscr{F}_{0}\right],
$$

where

$$
\begin{aligned}
\zeta_{n} & :=\sum_{i=1}^{K} b_{i} \nu_{n}^{i}, \\
W(n) & :=\sum_{m=0}^{n-1} W_{\nu_{m}+1}
\end{aligned}
$$

and $\nu_{i}^{n}$ is given by (3.25). 
In the remainder of the proof we follow Borovkov and Schassberger (1994). Unless stated otherwise we assume that $\rho \neq 1$, where

$$
\rho:=\sum_{i=1}^{K} \lambda_{i} b_{i} .
$$

Let $f: \mathbb{R}^{K} \rightarrow \mathbb{R}^{K}$ be the linear transformation given by

$$
f\left(e_{i}\right):=e_{i}+\frac{b_{i}}{1-\rho} \lambda, \quad i \in\{1, \ldots, K\},
$$

where $e_{i}$ is the $i$ th unit vector and the $i$ th component of $\boldsymbol{\lambda}$ is $\lambda_{i}$. We apply this transformation to obtain from (3.27) by a straightforward calculation that

$$
\begin{aligned}
E\left[f_{j}\left(\left(Y_{1}(n), \ldots, Y_{K}(n)\right)-\left(Y_{1}(0), \ldots, Y_{K}(0)\right)\right) \mid \mathscr{F}_{0}\right] \\
\quad=\frac{\lambda_{j}}{1-\rho} E\left[W(n) \mid \mathscr{F}_{0}\right]-E\left[\nu_{n}^{j} \mid \mathscr{F}_{0}\right], \quad j \in\{1, \ldots, K\},
\end{aligned}
$$

where $f_{j}$ is the $j$ th component of $f$.

Assume now that $\{\hat{X}(n)\}$ and hence also $\{Y(n)\}$ and $\left\{Y^{\prime}(n)\right\}$ are ergodic. Dividing both sides of (3.28) by $n$ and letting $n \rightarrow \infty$, the left-hand side tends to 0 and we obtain that

$$
\frac{\lambda_{j} \bar{w}}{1-\rho}=\bar{B}_{j} \pi_{j}, \quad j \in\{1, \ldots, K\} .
$$

In particular, we have $\rho<1$. Assume now that $\rho=1$. Dividing (3.27) by $n$ and letting $n \rightarrow \infty$, we obtain

$$
\bar{B}_{i} \pi_{i}=\lambda_{i} \bar{w}+\lambda_{i} \sum_{j=1}^{K} b_{j} \bar{B}_{j} \pi_{j} .
$$

Multiplying by $b_{i}$ and summing up yields an equation contradicting $\bar{w}>0$.

CoROLlary 3.7. If $\{\hat{X}(n)\}$ is ergodic, then (3.29) holds.

REMARK 3.8. Consider Example 2.1 or Example 2.2. Let $R(t)$ denote the residual walking time at time $t \in \mathbb{R}_{+}$if $S(t)=0$ and let $R(t)$ be the residual service time otherwise. Then $\left\{(X(t), R(t)): t \in \mathbb{R}_{+}\right\}$is a homogeneous Markov process. Using Theorem 3.1 and the properties of the Poisson process, it is not hard to prove that $\{(X(t), R(t))\}$ is Harris ergodic if (1.1) holds. Under natural additional assumptions this conclusion could also be made in the general case.

\section{APPENDIX}

This Appendix contains some results for discrete-time Markov processes and Markov chains. We consider a Markov process $X=\left\{X(n): n \in \mathbb{Z}_{+}\right\}$ taking values in the measurable space $(\mathbf{X}, \mathscr{X})$. The process $X$ is assumed to 
fulfill the following (standard) properties. The random elements $X(n)$ are defined on $(\Omega, \mathscr{F})$ and are measurable with respect to $\mathscr{F}_{n}$, where $\left\{\mathscr{F}_{n}: n \in \mathbb{Z}_{+}\right\}$ is an increasing sequence of $\sigma$-fields. For each $n \in \mathbb{Z}_{+}$there is a measurable mapping $\theta_{n}: \Omega \rightarrow \Omega$ such that $\theta_{m+n}=\theta_{m} \circ \theta_{n}$. Hence $\theta_{n}$ is the $n$th iteration of the shift operator $\theta:=\theta_{1}$. We assume that

$$
X \circ \theta_{m}(n)=X(m+n), \quad m, n \in \mathbb{Z}_{+},
$$

where the process $X \circ \theta_{m}$ is given by $X \circ \theta_{m}(n)(\omega):=X(n)\left(\theta_{m} \omega\right)$. We call $\theta_{n}$, $n \in \mathbb{Z}_{+}$, the family of shift operators associated with $X$. Further there is a family $P_{x}, x \in \mathbf{X}$, of probability measures on $(\Omega, \mathscr{F})$ such that $P_{x}(X(0)=x)=1$ and the mapping $x \rightarrow P_{x}(A)$ is measurable for all $A \in \mathscr{F}$ and such that, for any $\left\{\mathscr{F}_{n}\right\}$-stopping time $T$,

$$
P_{x}\left(X \circ \theta_{T} \in \cdot \mathscr{F}_{T}\right)=P_{X(T)}(X \in \cdot), \quad P_{x} \text {-a.s. on }\{T<\infty\},
$$

where we interpret all processes as random elements of the corresponding function space equipped with Kolmogorov's product $\sigma$-field. This is the strong Markov property of $X$. In particular,

$$
P_{y}\left(X \in \cdot \mid \mathscr{F}_{0}\right)=P_{x}, \quad P_{y} \text {-a.s. on }\{X(0)=x\}, \quad y \in \mathbf{X} \text {. }
$$

Assume that $\sigma$ is a finite $\{\mathscr{F}\}$-stopping time and define

$$
\sigma_{n+1}:=\sigma_{n}+\sigma \circ \theta_{\sigma_{n}}, \quad n \in \mathbb{Z}_{+},
$$

where $\sigma_{0}:=0$ and $\theta_{0}$ is the identity on $\Omega$. Then the $\sigma_{n}$ are stopping times and $Y(n):=X\left(\sigma_{n}\right), n \in \mathbb{Z}_{+}$, is again a Markov process in discrete time. One can take the same family $P_{x}, x \in \mathbf{X}$, of probability measures while the shift operator is given by $\theta_{\sigma}$ and the filtration by $\left\{\mathscr{F}_{\sigma_{n}}: n \in \mathbb{Z}_{+}\right\}$.

In the following $P$ denotes a probability measure on $(\Omega, \mathscr{F})$ of the form

$$
P(\cdot)=\int P_{x}(\cdot) \mu(d x),
$$

where $\mu$ is a probability measure on $(\mathbf{X}, \mathscr{X})$.

Under additional technical assumptions the conditions of the next lemma are known to imply the so-called geometric ergodicity. The proof of this as well as of the next result is not difficult and can follow along the lines of Meyn and Tweedie $(1993,1994)$ for example.

Lemma A.1. Assume that there are numbers $c \in(0,1)$ and $L \in \mathbb{R}_{+}$as well as a measurable function $V: \mathbf{X} \rightarrow \mathbb{R}_{+}$such that

$$
E\left[V(X(1)) \mid \mathscr{F}_{0}\right] \leq c V(X(0)) \quad \text { P-a.s. on }\{V(X(0))>L\} .
$$

Define

$$
\tau_{L}:=\min \{n \geq 1: V(X(n)) \leq L\},
$$

where $\min \varnothing:=\infty$. Then, for any $0<\delta \leq 1$,

$$
E\left[\sum_{n=0}^{\tau_{L}} V(X(n))^{\delta} \mid \mathscr{F}_{0}\right] \leq \frac{V(X(0))^{\delta}}{1-c^{\delta}}, \quad \text { P-a.s. on }\{V(X(0))>L\}
$$


and

$$
E\left[\tau_{L} \mid \mathscr{F}_{0}\right]<\infty, \quad P \text {-a.s. on }\{V(X(0))>L\} .
$$

Lemma A.2. Assume that the assumptions of Lemma A.1 hold and denote

$$
\gamma:=\min \{n \geq 1: V(X(n)) \leq c V(X(0))\} .
$$

Then, for any $0<\delta \leq 1$,

$$
E\left[\sum_{n=0}^{\gamma} V(X(n))^{\delta} \mid \mathscr{F}_{0}\right] \leq \frac{V(X(0))^{\delta}}{1-c^{\delta}}, \quad \text { P-a.s. on }\{V(X(0))>L / c\} .
$$

Moreover,

$$
E\left[\gamma \mid \mathscr{F}_{0}\right] \leq \frac{1}{(1-c) c}, \quad P \text {-a.s. on }\{V(X(0))>L / c\} .
$$

The next result is similar to Theorem 2.2 in Meyn and Tweedie (1994). For the reader's convenience we will present the proof.

Theorem A.3. Assume that there are numbers $c \in(0,1)$ and $L, c_{2}, d>0$, a measurable function $V: \mathbf{X} \rightarrow \mathbb{R}_{+}$and a finite $\left\{\mathscr{F}_{n}\right\}$-stopping time $\sigma \geq 1$ such that

$$
\begin{array}{rlrlrl}
\text { (A.4) } E\left[V(X(\sigma)) \mid \mathscr{F}_{0}\right] & \leq c V(X(0)), & & \text { P-a.s. on }\{V(X(0))>L\}, \\
\text { (A.5) } & E\left[\sigma \mid \mathscr{F}_{0}\right] & \leq c_{2} V(X(0)), & & \text { P-a.s. on }\{V(X(0))>L\}, \\
\text { (A.6) } & E\left[V(X(1)) \mid \mathscr{F}_{0}\right] & \leq d, & & \text { P-a.s. on }\{V(X(0)) \leq L\} .
\end{array}
$$

Then

$$
E\left[\tau_{L} \mid \mathscr{F}_{0}\right] \leq c^{\prime} \max \{V(X(0)), L\}, \quad \text { P-a.s. }
$$

for some finite $c^{\prime}$, where $\tau_{L}$ is given by (A.2). In particular, the set $\{x \in \mathbf{X}$ : $V(x) \leq L\}$ is positive recurrent, in the sense that

$$
\begin{aligned}
E\left[\tau_{L} \mid \mathscr{F}_{0}\right] & <c^{\prime} L, & \text { P-a.s. on }\{V(X(0)) \leq L\}, \\
\tau_{L} & <\infty, & \text { P-a.s. on }\{V(X(0))>L\} .
\end{aligned}
$$

Proof. Let $\sigma_{0}:=0$ and, recursively, $\sigma_{n+1}:=\sigma_{n}+\sigma \circ \theta_{\sigma_{n}}, n \geq 1$. Denoting

$$
\tau:=\min \left\{n \geq 1: V\left(X\left(\sigma_{n}\right)\right) \leq L\right\},
$$

we obtain from (A.5) that

$$
\begin{aligned}
E\left[\sigma_{\tau} \mid \mathscr{F}_{0}\right] & =E\left[\sum_{i=0}^{\tau-1} \sigma \circ \theta_{\sigma_{i}} \mid \mathscr{F}_{0}\right] \\
& =E\left[\sum_{i=0}^{\infty} \mathbf{1}\{i<\tau\} E\left[\sigma \circ \theta_{\sigma_{i}} \mid \mathscr{F}_{\sigma_{i}}\right] \mid \mathscr{F}_{0}\right] \\
& \leq c_{2} E\left[\sum_{i=0}^{\infty} \mathbf{1}\{i<\tau\} V\left(X\left(\sigma_{i}\right)\right) \mid \mathscr{F}_{0}\right] .
\end{aligned}
$$


By (A.4) we can apply Lemma A.1. Hence

$$
E\left[\sigma_{\tau} \mid \mathscr{F}_{0}\right] \leq \frac{c_{2}}{1-c} V(X(0)), \quad P \text {-a.s. on }\{V(X(0))>L\} .
$$

Since $\tau_{L} \leq \sigma_{\tau}$, this yields the assertion for $V(X(0))>L$. To prove the other case, we can use inequality $\tau_{L} \leq 1+\sigma_{\tau}^{\circ} \circ \theta_{1}$ for $V(X(1))>L$ to obtain, from (A.7),

$$
\begin{aligned}
E\left[\tau_{L} \mid \mathscr{F}_{0}\right] & =E\left[\mathbf{1}\{V(X(1)) \leq L\} \tau_{L} \mid \mathscr{F}_{0}\right]+E\left[\mathbf{1}\{V(X(1))>L\} \tau_{L} \mid \mathscr{F}_{0}\right] \\
& \leq P\left(V(X(1)) \leq L \mid \mathscr{F}_{0}\right)+E\left[\mathbf{1}\{V(X(1))>L\} E\left[\sigma_{\tau}^{\circ} \theta_{1}+1 \mid \mathscr{F}_{1}\right] \mid \mathscr{F}_{0}\right] \\
& \leq 1+\frac{c_{2}}{1-c} E\left[\mathbf{1}\{V(X(1))>L\} V(X(1)) \mid \mathscr{F}_{0}\right] .
\end{aligned}
$$

By assumption (A.6) the proof is complete.

Acknowledgments. This research has been motivated by the work of Rolf Schassberger. We would like to thank him for fruitful discussions. We would also like to thank Frank Kelly and Richard Tweedie for interesting comments and for drawing our attention to a class of greedy-type polling systems during a conference on applied probability in Oberwolfach.

\section{REFERENCES}

Altman, E. and Levy, H. (1994). Queueing in space. Adv. in Appl. Probab. 26 1095-1116.

BACCELl, F. and Foss, S. (1995). On the saturation rule for the stability of queues. J. Appl. Probab. 32 494-507.

Borovkov, A. A. (1994). Ergodicity and Stability of Stochastic Processes. TVP Publishers, Moscow. To appear.

Borovkov, A. A. and Schassberger, R. (1994). Ergodicity of a polling network. Stochastic Process. Appl. 50 253-262.

Coffman, E. G., JR. and Gilbert, E. N. (1987). Polling and greedy servers on a line. Queueing Systems Theory Appl. 2 115-145.

DAI, J. G. (1995). On positive Harris recurrence for multiclass queueing networks: A unified approach via fluid limit models. Ann. Appl. Probab. 5 49-77.

Fayolle, G. and Lasgouttes, J. (1994). A state-dependent polling model with Markovian routing. INRIA Report 2279.

Fayolle, G., Malyshev, V. A. and Menshikov, M. V. (1995). Topics in the Constructive Theory of Countable Markov Chains. Cambridge Univ. Press.

Foss, S. and Chernova, N. (1996). Ergodic properties of polling systems. Problems Inform. Transmission. To appear.

Foss, S. and LAST, G. (1995). On the stability of greedy polling systems with general service policies. Preprint.

FrickeR, C. and JAIBI, M. R. (1994). Monotonicity and stability of periodic polling systems. Queueing Systems Theory Appl. 15 211-238.

GEORgIAdis, L. and SzPANKowski, W. (1992). Stability of token passing rings. Queueing Systems Theory Appl. 11 7-34.

Kroese, D. P. and Schmidt, V. (1992). A continuous polling system with general service times. Ann. Appl. Probab. 2 906-927.

Kroese, D. P. and Schmidt, V. (1994). Single-server queues with spatially distributed arrivals. Queueing Systems Theory Appl. 17 317-345. 
Last, G. and Brandt, A. (1995). Marked Point Processes on the Real Line: The Dynamic Approach. Springer, New York.

Levy, H., SidI, M. and Boxma, O. J. (1990). Dominance relations in polling systems. Queueing Systems Theory Appl. 6 155-171.

Malyshev, V. A. and Menshikov, M. V. (1982). Ergodicity, continuity and analyticity of countable Markov chains. Trans. Moscow Math. Soc. 1 1-48.

MAssoulié, L. (1995). Stability of non-Markovian polling systems. Queueing Systems Theory Appl. 21 67-95.

Meyn, S. P. and Tweedie, R. L. (1993). Markov Chains and Stochastic Stability. Springer, London.

Meyn, S. P. and Tweedie, R. L. (1994). State-dependent criteria for convergence of Markov chains. Ann. Appl. Probab. 4 149-168.

SCHASSBERGER, R. (1993). Stability of polling networks with state-dependent server routing. Technical Report 12/93, Technical Univ. Braunschweig.

TAGAKI, H. (1990). Queueing analysis of polling systems. In Stochastic Analysis of Computer and Communication Systems (H. Takagi, ed.) 267-318. North-Holland, Amsterdam.

NOVOSIBIRSK STATE UNIVERSITY

630090 NovosiBIRSK

Pirogova 2

RUSSIAN FEDERATION

E-mail: FOSS@math.nsk.su
INSTITUT FÜR MATHEMATISCHE StOCHASTIK TEChNische UnIVERSitÄT BRAUNSCHWEIG

Pockelsstrasse 14, Postfach 3329

38023 BRAUNSCHWEIG

GERMANY

E-mail: G.Last@tu-bs.de 